This item was submitted to Loughborough's Research Repository by the author.

Items in Figshare are protected by copyright, with all rights reserved, unless otherwise indicated.

\title{
Phase transitions to dipolar clusters and charge density waves in high Tc superconductors
}

\section{PLEASE CITE THE PUBLISHED VERSION}

http://dx.doi.org/10.1016/j.physc.2016.08.007

\section{PUBLISHER}

(c) Elsevier

VERSION

AM (Accepted Manuscript)

\section{PUBLISHER STATEMENT}

This work is made available according to the conditions of the Creative Commons Attribution-NonCommercialNoDerivatives 4.0 International (CC BY-NC-ND 4.0) licence. Full details of this licence are available at: https://creativecommons.org/licenses/by-nc-nd/4.0/

\section{LICENCE}

CC BY-NC-ND 4.0

\section{REPOSITORY RECORD}

Saarela, M., and F.V. Kusmartsev. 2019. "Phase Transitions to Dipolar Clusters and Charge Density Waves in High Tc Superconductors”. figshare. https://hdl.handle.net/2134/22439. 


\title{
Phase transitions to dipolar clusters and charge density waves in high $\mathrm{T}_{c}$ superconductors
}

\author{
M. Saarela ${ }^{\mathrm{a}}$, and F. V. Kusmartsev ${ }^{\mathrm{b}}$ \\ ${ }^{a}$ Department of Physics, P.O. Box 3000, FIN-90014, University of Oulu, Finland \\ ${ }^{b}$ Department of Physics, Loughborough University, LE11 3TU, UK
}

\begin{abstract}
We show that doping of hole charge carriers leads to formation of electric dipolar clusters in cuprates. They are created by manybody interactions between the dopant ion outside and holes inside the $\mathrm{CuO}$ planes. Because of the two-fold degeneracy holes in the $\mathrm{CuO}$ plane cluster into four-particles resonance valence bond plaquettes bound with dopant ions. Such dipoles may order into charge-density waves (CDW) or stripes or form a disordered state depending on doping and temperature. The lowest energy of the ordered system corresponds to a local anti-ferroelectric ordering. The mobility of individual disordered dipoles is very low at low temperatures and they prefer first to bind into dipole-dipole pairs. Electromagnetic radiation interacts strongly with electric dipoles and when the sample is subjected to it the mobility changes significantly. This leads to a fractal growth of dipolar clusters. The existence of electric dipoles and CDW induce two phase transitions with increasing temperature, melting of the ordered state and disappearance of the dipolar state. Ferroelectricity at low doping is a natural consequence of such dipole moments. We develop a theory based on two-level systems and dipole-dipole interaction to explain the behavior of the polarization as a function of temperature and electric field.
\end{abstract}

Keywords: high Tc superconductors, charge density wave, pseudogap, dipolar cluster

PACS: 74.72.Gh, 74.72.Kf

\section{Introduction}

Since the discovery of the hole-doped cuprate superconductors a great deal of effort has been devoted to understand their complex behaviour. Recently guided migration of doped oxygen atoms in $\mathrm{La}_{2} \mathrm{CuO}_{4+y}$ (LCO) has been successfully demonstrated with nano-scale synchrotron radiation scanning $\mathrm{X}$-ray diffraction in the temperature range $180 \mathrm{~K}<\mathrm{T}<330 \mathrm{~K}$. [1, 2, 3] Originally randomly distributed dopant atoms in the $\mathrm{LaO}$ plane order into clusters after many hours of illumination. Moreover, these ordered nano grains grow and form a fractal structure that extends through the sample. The cluster growth looks as if there is a continuous phase transition (or a critical state) where there are two competing long-range orders(LRO). There exists a potential barrier separating minima associated with these orders and it takes a time for the dopant oxygens to self-organize and reach the new stable ordered phase. Probably because of this criticality the cluster sizes observed have a scale free distribution. The superconducting critical temperature depends on the type of the cluster order. There are two critical temperatures of the clusters LRO observed. It was found that the higher transition temperature is associated with the better order where the critical percolation through ordered oxygens takes place. These fundamental discoveries [1, 2, 3] pose questions: Why does the radiation increase mobility of oxygen ions and why do they order? Is this order critical in increasing the onset temperature to superconductivity?

The inhomogeneity of cuprate superconductors coupled with dopant atoms is well documented besides LCO also in other materials and in different experiments using scanning tunneling spectroscopy [4, 5, 6, 7, 8], photoemission spectra (ARPES) [9] - suggesting that the oxygen dopant-induced states are mixed with $\mathrm{Cu}$ atoms - and measurements of dielectric constant [10] etc.

Very recently a surprising discovery of ferroelectricity also in $\mathrm{La}_{2} \mathrm{CuO}_{4+y}$ was reported at exceptionally low oxygen doping. $[11,12]$ To describe this effect the Ginsburg-Landau theory of the ferroelectricity, where the existence of the magneto-electric coupling has been assumed, was developed. [13] Other models based on polaron fromation and vortexantivortex pairs have been suggested. Here we propose that it is more natural to assume that the ferroelectricity arises due to electric dipoles caused by the Coulomb interaction of the dopant ions.

Debate between two competing orders and preformed pairs of superconductivity in the pseudogap region focuses mainly in the interpretation of ARPES measurements on the nature of gap functions and Fermi arcs. [14, 15, 16, 17, 18, 19] Very recent experiments found evidences of sharp phase transitions at temperatures between the superconducting critical temperature $\mathrm{T}_{c}$ and the pseudogap temperature at $\mathrm{T}^{*}$. [20, 21, 22, 23] At zero temperature inside the superconducting dome two phase transitions are discovered by studying the behavior of the gap function as a function of doping. [24] Also the temperature dependence of resistivity changes from quadratic to linear behavior inside the pseudogap region with increasing doping and temperature. $[25,26]$ Optically stimulated ultrafast changes in the charge-density wave correlations have been studied by femtosecond resonant $\mathrm{x}$-ray diffraction indicating that charge order- 
ing and superconductivity are competing orders.[27] However, in spite of many attempts there is still no clear physical mechanism of the pseudogap and why there are phase transition between the pseudogap temperature $\mathrm{T}^{*}$ and $\mathrm{T}_{c}$.

Important experimental findings for our model come from a peculiar temperature dependence of the Hall coefficient, $\mathrm{R}_{H}$, measured in $\mathrm{Sr}$ doped $\mathrm{La}_{2-x} \mathrm{Sr}_{x} \mathrm{CuO}_{4}$ (LSCO) [28, 29]. These results have been analyzed within a two-band model [30]. It was argued that the charge carrier density, in that case the hole density, can be written as the sum of two components

$$
n_{\mathrm{h}}(x, T)=n_{0}(x)+n_{1} e^{-\Delta(x) / 2 k_{B} T} .
$$

The first term depends on doping $x$ and is independent of temperature $T$. The second term is of activation type contribution with a doping dependent activation energy $\Delta(x)$ multiplied by a constant $n_{1}$. The analysis [30] suggested that at small doping, $0.01<x<0.08$, and below the room temperature each dopant atom creates one hole and hence $n_{0}(x)=x$. At higher temperatures the hole density increases rapidly and for each $x$ that temperature behavior was well fitted with the activation type exponential component in Eq. (1). The constant value $n_{1}=2.8$ indicated that more than one hole was activated. Even larger value $n_{1} \approx 4$ was found by Ono et al. [29]. The true nature of this two-component behaviour and the activation energy $\Delta(x)$ was left as an open question. At higher doping $0.08<x<0.21$ and below the room temperature the hole density increases as a function of doping much faster than the number of dopant atoms and, more strangely, for a given $x$ when the temperature increases from $0 \mathrm{~K}$ to $50 \mathrm{~K}$ the carrier density decreases. This suggests that the charge density can fluctuate between the two bands.

In this paper we propose a microscopic approach [33] where bound states appear because dopant atoms (e. g. $\mathrm{Sr}^{2+}$ are replacing the $\mathrm{La}^{3+}$ ) and therewith inducing extra negative charges into spacer layers. At low doping these negative charges are able to bind holes. The key ingredient in binding holes into dopant atom is the near degeneracy of the d-state bands with $x^{2}-y^{2}$ and $z^{2}$ symmetry in the $\mathrm{CuO}$ plane. The Jahn-Teller distortions in cuprates, such as $\mathrm{La}_{2} \mathrm{CuO}_{4}$, elongate the oxygen octahedron (green in Fig.1) surrounding the central $\mathrm{Cu}$ ion and split the degeneracy of the associated $e_{g}$ orbitals of the $\mathrm{Cu} d^{9}$ state. This happens in the highest partially occupied $x^{2}-y^{2}$ - orbital, which together with the doubly occupied $\mathrm{O} p_{x}, p_{y^{-}}$ orbitals form a strong covalent bonding. Their straight hybridization gives rise to the bonding, non-bonding and halffilled anti-bonding bands and predicts a good metal, in sharp contrast with experiments finding a large charge gap. The failure of the band theory indicates a strong Coulomb interaction (in particular, on site Hubbard $U$ ), which may exceed well the bandwidth of the tight-binding, anti-bonding band.

If we take the parent compound as $\mathrm{La}_{2} \mathrm{CuO}_{4}$ then two charge carriers (holes) put into the $\mathrm{Cu} x^{2}-y^{2}$ orbital would experience a large energy penalty, $(U \sim 10 e V)$. Since on site Hubbard $U$ is much larger than the energy separation between the $\mathrm{Cu}$ $x^{2}-y^{2}$ and $\mathrm{O} p$-orbitals, which defines the charge transfer gap $\Delta_{C T} \sim 1-2 \mathrm{eV}$, and this in turn is much larger than the hybridisation integral, $t_{d p} \sim 0.350 \mathrm{meV}[34,35,36]$, single electrons

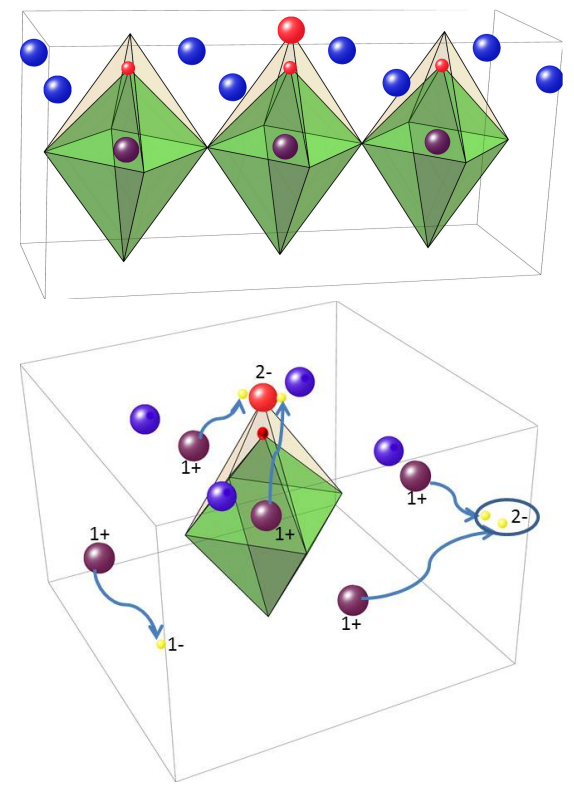

Figure 1: Parts of the $\mathrm{La}_{2} \mathrm{CuO}_{4}$ crystal structure with one dopant impurity (topmost red sphere) are shown in two panels. Green octahedra have oxygens in all corners and $\mathrm{Cu}$ atom at the center (purple sphere). The apex oxygens (red spheres) and $\mathrm{La}$ atoms (blue spheres) form one of the two $(\mathrm{LaO})^{1+}$ spacer layers, which separates the $\left(\mathrm{CuO}_{2}\right)^{2-}$ planes. In the upper panel we show how dopant impurity atom with its negative charge pushes the apex oxygen down from the top of light red octahedron to the top of the green octahedron. This restores the $e_{g}$ symmetry of the $\mathrm{Cu}$ orbitals (the anti-Jahn-Teller effect), which releases electrons from $\mathrm{Cu}$ atoms. Note, that there original ionic states on $\mathrm{Cu}^{2+}$ have small positive charge transfer gap $\Delta_{C T}$. But the formal valence state $\mathrm{Cu}^{3+}$, which should be created by doping, have large negative $\Delta_{C T}<0$. This means that the states should be rather represented as $\mathrm{Cu}^{3+} \rightarrow \mathrm{Cu}^{2+} L$, (see, the Ref. [31] for a detail), where the doped holes would go not so much to the d-shells but rather to the oxygens, creating oxygen holes (though the quantum numbers of the respective states would be the same as those of $\mathrm{Cu}^{3+}$ ). In the lower panel we show schematically how electrons are redistributed. Thus, each $\mathrm{Cu}$ (precisely saying the $\mathrm{Cu}$ atom with surrounding oxygens) can "loose" effectively one electron. Two of them are captured by the impurity oxygen to fill its p-shell. This extra charge repels nearest neighbour $\mathrm{Cu}$ electrons and altogether five holes are revealed. The degeneracy allows four holes to bind into the ground state of the impurity charge 2 - and form a dipolar cluster.

are localized on $\mathrm{Cu}$ sites on the $\mathrm{Cu} x^{2}-y^{2}$ orbitals forming the $\mathrm{Cu}^{2+}$ states. Their spins are anti-ferromagnetically aligned (via the super-exchange interaction that involves virtual hopping to the neighbouring $\mathrm{O} p$ - orbitals) to create the anti-ferromagnetic Mott insulator[37].

Since these original ionic states on $\mathrm{Cu}^{2+}$ have positive charge transfer gap $\Delta_{C T}$, which is small relatively to $U$, the formal valence state $\mathrm{Cu}^{3+}$, which with doping would already have large negative $\Delta_{C T}<0$ should be rather represented by Zhang-Rice singlet, i.e. as $\mathrm{Cu}^{3+} \rightarrow \mathrm{Cu}^{2+} L$, (see, the Ref. [31] for details). There doped holes would go not so much to the d-shells but rather to the oxygens, creating oxygen holes (though the quantum numbers of the respective states would be the same as those of $\mathrm{Cu}^{3+}$ ). In these Zhang-Rice singlets the hole is distributed between the $\mathrm{Cu}^{3+}$ and four neighbouring oxygens. Having this in mind to make it short below we call these Zhang-Rice singlets simply saying as $\mathrm{Cu}^{3+}$ states.

In our approach we consider this Mott state and take into ac- 
count that cuprates, like $\mathrm{La}_{2} \mathrm{CuO}_{4}$, are also layered ionic crystals. The $\left(\mathrm{CuO}_{2}\right)^{2-}$ layer is negatively charged and two spacer layers $\left(\mathrm{La}_{2} \mathrm{O}_{2}\right)^{2+}$ are positively charged. Oxygen atoms form an octahedral cell around $\mathrm{Cu}^{2+}$ and the apex oxygen atoms containing two extra electrons filling the p-shell are in the LaOlayer as depicted in Fig. 1. In the parent anti-ferromagnetic insulator compound the degeneracy of cubic $e_{g}$ states is removed by the Jahn-Teller effect, which elongates the oxygens octahedron. Copper, on the other hand, is a transition metal and it can easily give out one electron and transfer into $\mathrm{Cu}^{3+} \rightarrow \mathrm{Cu}^{2+} L$ sharing the electron with neighbouring octahedron oxygens and forming the Zhang-Rice singlet.

Dopant atoms settle in the $\mathrm{LaO}$ spacer layer substituting $\mathrm{La}$ atoms like in the case of $\mathrm{Sr}$ doping or intercalated like oxygen atoms. Oxygen atoms are small in size and thus mobile. Surprisingly that these atoms play an important role in the vortex trapping[32]. They easily fill their p-shell with two electrons and become ionized. When this happens the negative impurity charge pushes the negatively charged apex oxygen down (see, Fig.1). This apex atom displacement restores the $e_{g}$ symmetry and helps to remove one electron from neighbouring $\mathrm{Cu}$-ions to form Zhang-Rice singlet (the anti-Jahn-Teller effect). ${ }^{1}$ At large enough doping holes created in this way in $\mathrm{Cu}$ sites form a charge carrying hole ("Zhang-Rice singlet") band in the $\mathrm{CuO}$ plane. Many complexes containing specific transition-metal central ions with special valency show this effect. [39] Every new hole appearing during hole-doping in the parent compound leads to strong frustration of the original antiferromagnetic state. This leads to rapid suppression of antiferromagnetism in $\mathrm{La}_{2-x} \mathrm{Sr}_{x} \mathrm{CuO}_{4}$ even by small hole concentration.

\section{Charge Density Waves composed of resonance plaque- ttes and electrical dipolar moments}

Four holes bound by dopant atoms due to correlations [40] form resonance plaquettes in a manner similar to the resonance valence bond described by Anderson[37]. These plaquettes together with dopant atoms induce electrical dipolar moments. There may also arise a second nearly flat band[38].

As discussed above in hole doped cuprates dopant atoms are located in spacer layers between $\mathrm{CuO}$ planes and charge carriers are confined within $\mathrm{CuO}$ planes. Dipolar clusters exist in a broad range of doping from deeply under-doped up to over-doped region. They disappear in over-doped region due to screening associated with the increasing hole density. In the region of the optimal doping plaquettes are decoupled from dopand atoms and become mobile. Then they are playing a key role in the Planckian dissipation observed in all cuprates[41]. The dipole-dipole interaction between clusters has a strong directional dependence. It is attractive when parallel dipoles pointing to the same direction are on top of each other and repulsive when they are side by side.

\footnotetext{
${ }^{1}$ Note that with this anti-Jahn-Teller effect associated with the squeezing of the oxygen octahedra, the hole state has a two fold degeneracy that is in addition of the conventional Kramers degeneracy.
}

Dopant oxygen atoms are mobile although their mobility is very low at low temperatures. Then at temperatures below 80 $\mathrm{K}$, at very low doping and random distribution of dopant atoms it is likely that dipoles remain in random, glassy order. Mobility rises with temperature and when the sample is subjected to electromagnetic (X-ray) radiation. The radiation interacts strongly with electric dipoles as well as doped oxygen impurities. They can be excited by irradiation and moved to new positions associated with the minimum of the total energy. That is how irradiation increases the effective mobility of dopant atoms and ordering of dipoles. As the result they reorganize themselves with the help of the dipole-dipole interaction and after waiting long enough, as it was done in Refs. [1, 2], a short-ranged order arises.

The interaction between dipolar clusters is short-ranged and varies as a function of separation distance with the $1 / r^{3}$ tail. Clusters move towards the minimum energy configuration, but, because of low mobility, nucleation can start at different parts of the sample and lead to patches of different directional stripy order with a fractal structure like in classical dipolar systems. The electron-phonon interaction will further enhance this ordering tendency leading to charge density waves[42, 43, 44].

Any isolated charge associated with an impurity or a charge fluctuation in $\mathrm{LaO}$ spacer layer is inducing the anti-Jahn-Teller shift of the apex $\mathrm{O}$ ion[33, 40] and therewith creates a resonance plaquette in $\mathrm{CuO}$ plane. To describe these resonance plaquettes we developed the many-body variational theory where four holes are trapped by the impurity [33, 45, 46, 47] and derived a proper energetic description of resonance plaquette states, which reproduces experimental pseudogap temperature within the two-fluid model [30, 29].

\section{Many-body theory of the dipole moment formation}

In order to describe properties of a single dipole we need to solve a quantum many-body problem where holes interact via long-ranged Coulomb interaction among themselves and with the isolated charge associated with an impurity inducing the anti-Jahn-Teller shift of the apex $\mathrm{O}$ ion [33, 40, 48]. In fact the many body interaction results in the bound state of the impurity in the $\mathrm{LaO}$ plane with holes in the $\mathrm{CuO}$ plane. We calculate below the binding energy of holes forming the dipole as a function of doping. To make a quantitative description of such a state it is sufficient to use a continuum approximation, where mobile charged holes are confined to a $\mathrm{CuO}$ plane. Their effective mass is determined by the band structure of the material with the use of the effective mass or the (kp-) method [49]. For a simplicity, one may start with a three band Hubbard model[31,50] where the effective holes mass, $m \sim t_{p d}^{-1}$. The charge neutrality is implemented by the inert background, called the jellium model[33]. Holes are allowed in general to move in a 2D-plane and the impurity is set at a given distance $c$ away from the plane. Thus the impurity serves as a nucleation center of the dipole moment pointing to c-direction.

The Hamiltonian of such a system is the sum of two terms, $H=H_{h}+H_{I}$ which includes the mutual Coulomb interaction 
and kinetic energy of holes, $H_{h}$ and the apex $\mathrm{O}$ ion interaction with holes, $H_{I}$,

$$
\begin{aligned}
& H_{h}=-\sum_{i=1}^{N} \frac{\hbar^{2}}{2 m} \nabla_{i}^{2}+\frac{1}{2} \sum_{i, j=1, i \neq j}^{N} \frac{e^{2}}{4 \pi \epsilon\left|\mathbf{r}_{i}-\mathbf{r}_{j}\right|} \\
& H_{I}=-\frac{\hbar^{2}}{2 M} \nabla_{0}^{2}-\sum_{i=1}^{N} \frac{2 e^{2}}{4 \pi \epsilon \sqrt{\left|\mathbf{r}_{0}-\mathbf{r}_{i}\right|^{2}+c^{2}}} .
\end{aligned}
$$

The number of mobile holes is $N$, they have the mass $m$, charge $|e|$ and position $\mathbf{r}_{i}$. The impurity is placed at $\mathbf{r}_{0}$, its charge is $-2|e|$ and kinetic energy is controlled by the mass $M$. For a localized impurity we let the mass grow to infinity. The strength of the Coulomb interaction depends on the dielectric constant $\varepsilon$. It is convenient to use the atomic units where all distances are given in units of $r_{0}=r_{s} r_{B}$ and energies in Rydbergs. The parameter $r_{s}=1 / \sqrt{\pi n_{0}} r_{B}$ is defined by the density $n_{0}$ of holes and the Bohr radius $r_{B}$.

At low doping in the under-doped region the hole gas is dilute, but very strongly correlated. We assume that the hole band is two-fold degenerate, because the mechanism to produce the conducting hole band relies on the anti-Jahn-Teller type shift in almost degenerate energy levels. Under such circumstances, in strongly correlated regime, the difference between the fermionic and bosonic gases is of minor importance. The cluster formation takes place in both cases although in slightly different regions of $r_{s}$ values [46, 47]. The bosonic part of the ground-state wave function which contains correlations between holes and impurities is chosen in the form of the Jastrowtype variational ansatz[51]

$$
\begin{aligned}
\Psi & =e^{\frac{1}{2} \sum_{i, j=1}^{N} u^{h h}\left(\left|\mathbf{r}_{i}, \mathbf{r}_{j}\right|\right)} \\
\Psi_{I} & =e^{\frac{1}{2} \sum_{i=1}^{N} u^{I t}\left(\left|\mathbf{r}_{i}, \mathbf{r}_{0}\right|\right)} e^{\frac{1}{2} \sum_{i, j=1}^{N} u^{I n}\left(\left|\mathbf{r}_{i}, \mathbf{r}_{0}\right|\right)} \Psi
\end{aligned}
$$

We have extended the conventional, many-body variational theory to the case where four holes are trapped by the impurity.[33] The wave function includes now the product of three components, hole-hole (hh) correlations, impurity-trapped holes (It) correlations and impurity non-trapped holes (In) correlations. The correlation functions $\left.\left.u^{h h}\left(\left|\mathbf{r}_{i}, \mathbf{r}_{j}\right|\right)\right), u^{I t}\left(\left|\mathbf{r}_{i}, \mathbf{r}_{0}\right|\right)\right)$ and $\left.u^{I n}\left(\left|\mathbf{r}_{i}, \mathbf{r}_{0}\right|\right)\right)$ are determined by minimizing the total energy $E$ of the hole gas and the chemical potential $\mu$ of the impurity.

$$
\begin{aligned}
E & =\frac{\left\langle\Psi\left|H_{h}\right| \Psi\right\rangle}{\langle\Psi \mid \Psi\rangle} \\
\mu & =\frac{\left\langle\Psi_{I}\left|H_{I}\right| \Psi_{I}\right\rangle}{\left\langle\Psi_{I} \mid \Psi_{I}\right\rangle}-\frac{\left\langle\Psi\left|H_{h}\right| \Psi\right\rangle}{\langle\Psi \mid \Psi\rangle}-4 E_{\text {bin }},
\end{aligned}
$$

where the last term is the sum of binding energies $4 E_{\text {bin }}$ of four trapped holes. The maximum number of trapped holes in the same state is determined by the Pauli principle. It is twice the degeneracy factor of the hole band, which we set equal to two. The fact that four holes are bound by the charge $-2|e|$ is caused by strong many-body correlations. As a result of the variational calculation we get the total and binding energies, chemical potential and the hole-hole and hole-impurity distribution functions. For details we refer to Ref. [33].

In Fig. 2 we show the binding energy $E_{b i n}$ as a function of $r_{s}$ parameter. The binding energy vanishes when $r_{s}<2.5$ and

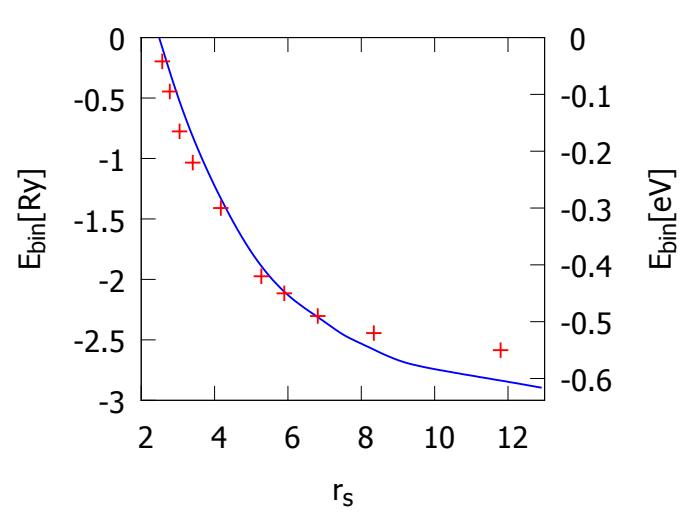

Figure 2: The calculated binding energy of the hole cluster $E_{b i n}(x)$ (solid blue curve) identified as the activation energy and fitted to the Hall coefficient measurements [29, 52] (red plus signs).

saturates very slowly to the single hole limit when $r_{s} \rightarrow \infty$. At zero temperature the number of holes in $\mathrm{CuO}$ layers is equal to $x$, the number of dopant atoms, but when the density is high enough, $r_{s}<2.5$, then four more holes per dopant atom are released. The maximum number of holes is one per Copper site and that is reached when $x=1 / 5=0.2$. This is clearly seen by Cooper et al. [25] as a change in the temperature dependence of the resistivity at $\mathrm{x} \approx 0.19$, which is identified as a quantum critical point. In the Gorkov-Teitelbaum two-fluid model[30] the activation energy $\Delta(x)$ vanishes also at $x \approx 0.2$.

The cluster formation gives the microscopic foundation to the assumption of two kinds of charge carries, doped and activated. In order to allow the transfer of holes between these two components seen in the Hall coefficient data [29] we have refitted them with the formula,

$$
n_{h}=x(1+4 * f(x))+4(1-f(x)) e^{-\Delta(x) /\left(2 k_{B} T\right)}
$$

The function $f(x)$ shown in Fig. 3 gives the fraction of holes freed from the clusters already at zero temperature. In an ideal system $f(x)$ should be a step function, zero at low doping and one when all holes are released from the trap. At zero temperature that happens at $x=0.2$ and the density of holes per $\mathrm{Cu}$ site $n_{h}=1$. That is the phase transition from dipolar cluster state to homogeneous metallic state. In practice high $\mathrm{T}_{c}$ superconductors are inhomogeneous. There are high and low density regions and that is seen as a smooth behavior in the fitted $f(x)$. In the high density regions, $r_{s}<2.5$, the binding energy of clusters vanishes and all four bound holes per cluster are then free to carry the charge and out of the activation process. In the fit we have averaged the low temperature fluctuations between $0 \mathrm{~K}<T<50 \mathrm{~K}$. In Fig. 3 we show the fitted values. For low doping, $x<0.08, f(x)$ vanishes and then increases up to 0.6 at the highest doping, $\mathrm{x}=0.21$, measured. Taking strictly the zero temperature limit of experiments the values of $f(x)$ grow faster with increasing doping reaching unity when $x=0.2$, which indicates that all holes are freed.

The fitted activation energies $\Delta(x)$ shown in Fig. 2 by red symbols can be compared with our binding energies $-E_{b i n}\left(r_{s}\right)$ after the conversion of our Rydberg unit into electron volts and 


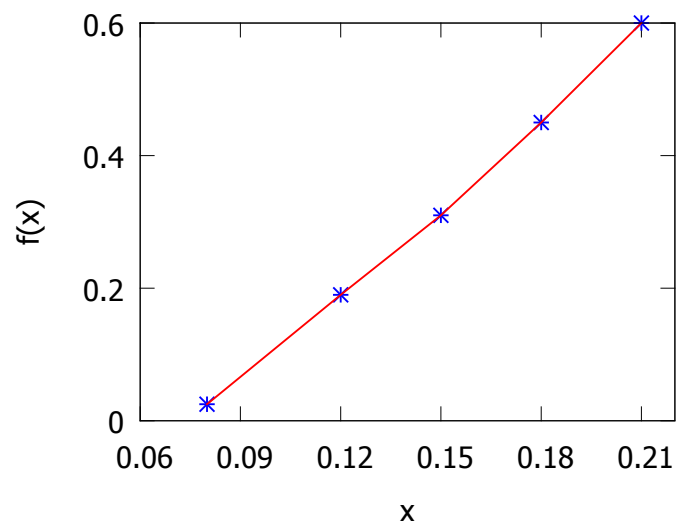

Figure 3: The function $f(x)$ from Eq. (5) fitted to the Hall coefficient data by Ono el al. [29] at values of doping show by blue stars. The solid line is just to guide the eye.

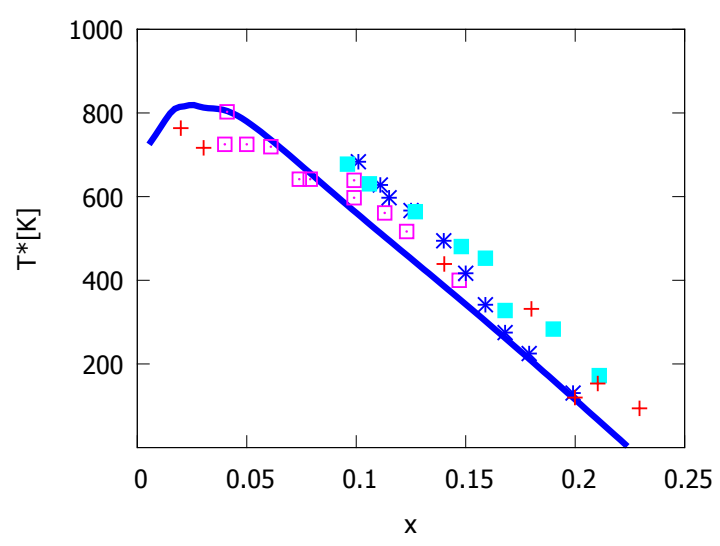

Figure 4: The pseudogap temperature calculated from Eq. (7) is compared with the crossover temperature of the resistivity curves (open squares)[[54]], the temperature of the maximum magnetic susceptibility (stars) [[55] ] and (solid squares) [ [56]], and with the activation energy (plus-signs)[[30]].

$r_{s}$ parameter scale into the doping scale $x$. In our notation the density of holes $n_{h}=1 / \pi\left(r_{s} r_{B}\right)^{2}=x / \pi r_{\mathrm{Cu}}^{2}$ where $r_{\mathrm{Cu}} \approx 1.9 \AA$ is half of the separation distance between Copper atoms. We identify the point $r_{s}=2.5$ where $E_{b i n}(x)$ vanishes with the value of doping $\mathrm{x}=0.22$ which is slightly higher than the value 0.2 where the activation energy vanishes because of the fluctuations as pointed out above. That fixes the Bohr radius $r_{B} \approx 1.64 \AA$. Then we are left with one parameter to convert the energy scale. By choosing $1 \mathrm{Ry}=0.213 \mathrm{eV}$ we get the fit shown in Fig. 2 by blue solid curve. Taking into account these values of Rydberg and Bohr radius we get the dielectric constant $\epsilon=21$ and the effective hole mass $m=6.7 m_{e}$ in units of the electron mass $m_{e}$. The dielectric constant found here is in reasonable agreement with the value $\epsilon=29$ determined from Chen et al. experiments in LSCO [53].

We identify the pseudogap temperature with the temperature when all trapped holes are released. Then

$$
x=e^{-\Delta(x) /\left(2 k_{B} T^{*}\right)},
$$

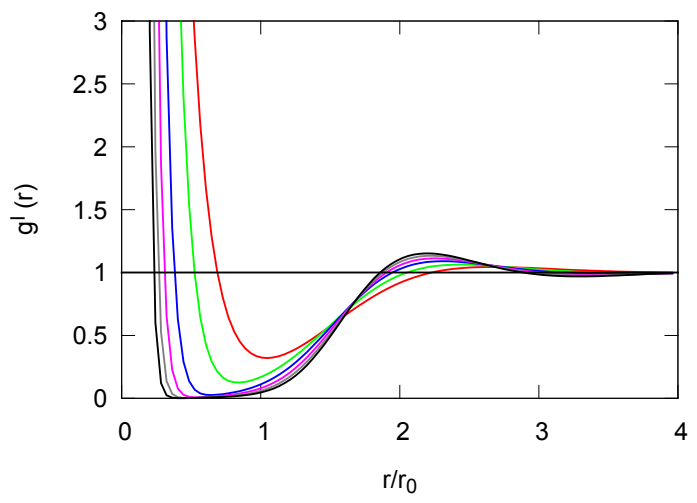

Figure 5: The radial distribution functions of holes in the $\mathrm{CuO}$ layer around the impurity charge $-2|e|$ in the $\mathrm{LaO}$ layer. The impurity is located at the origin and the peak around it is huge in this scale. The highest peak is at the lowest density when $r_{s}=12$ with the height 780 . The curves from left to right are calculated at $r_{s}=12,10,8,6,4$ and 2.5 , respectively. All distributions are normalized to unity at $r \rightarrow \infty$. The deep minima at $r<1$ separates four bound holes from the continuum holes.

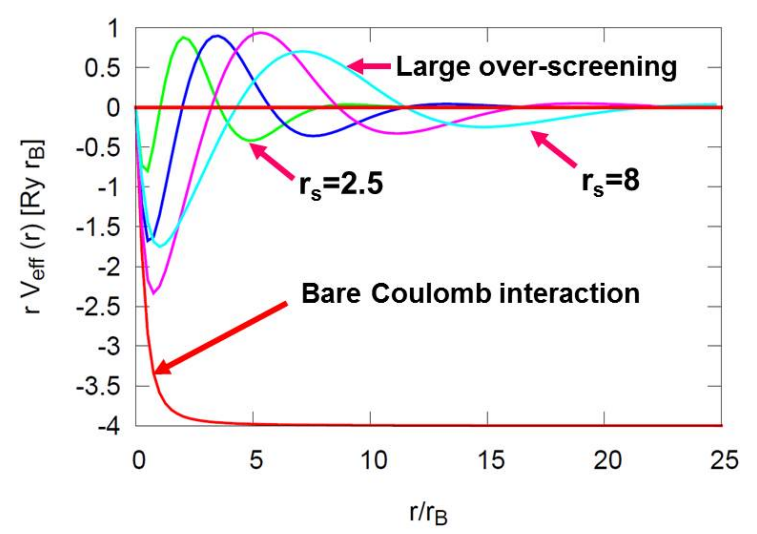

Figure 6: The hole-impurity effective interaction as a function of separation distance $r$ and multiplied by $r$ calculated at $r_{s}=8,6,4$ and 2.5 as marked in the figure. Also shown is the bare Coulomb interaction with the red curve at the bottom.

and the density of holes $n_{h}=5 x$. That is the phase transition line in temperature doping plane when dipolar clusters melt into the bad metal state. This transition is not sharp because of the density fluctuations, and one enters the Fermi liquid regime with regions of clusters. That would be some kind of an emulsion state. In Fig. 4 we show the calculated pseudogap temperature

$$
T^{*}(x)=E_{\mathrm{bin}}(x) /\left(2 k_{B} \log (x)\right)
$$

and find a good agreement with different experimental values. Doping dependence of $T^{*}(x)$ is dominated by the binding energy because $\log (x)$ is a slowly varying function in the range $0.05<x<0.2$.

In Fig. 5 we show the radial distribution of holes in $\mathrm{CuO}$ plane around an impurity, which is located at half a Bohr radius above the $\mathrm{CuO}$ plane. The bound trapped holes correspond to a large peak in $g^{I}(r)$ around the origin. At the highest density 
where the activation energy vanishes $\left(r_{s}=2.5, r_{0}=4.1 \AA\right.$ and $x=0.22)$ the radius of the bound state wave function barely covers the nearest neighbour $\mathrm{Cu}$ atoms, since the distance between $\mathrm{Cu}$ atoms is about $3.8 \AA$. When the hole density decreases the range of wave function extends further covering the whole neighbouring octahedron, see, the Fig. 1 and the yellow circle in the Fig. 7. As pointed out earlier the Pauli principle allows only four particles to be bound in the lowest state and that is why one of the five covered holes (the maximum number of holes) is free to act as a charge carrier. That is consistent with the experimental fact that the number of holes is equal to the number of dopant atoms at low doping and the maximum hole density is reached when all five holes per dopant atom are released.

Finally in Fig. 6 we show the effective interaction between the impurity and holes. At short distances it is attractive, but many body effects strongly over-screen attraction at intermediate ranges leading to bound clusters.

Four bound, positively charged holes over-screen the impurity ion charge $-2|e|$. It means that two electrons are expelled from the octahedron as circled out in Fig. 1. They are attracted to the positively charged LaO-layer and get loosely trapped by four apex oxygens squeezed to their symmetry positions in the same way as the impurity ion does. That is a new source of electron pairs, which will form a very flat band together with the bound holes in the $\mathrm{CuO}$ plane. The binding energy of holes to the electron charges is roughly the same as to the impurity charge and that is why at low doping the factor in front of the activation contribution to the charge carriers is independent of $x$ in Eqs. (1) and (5). This phenomenon is called Mottness. In Refs. [57, 58], however, a different microscopic mechanism for the Mottness accounting for spectral weight transfer experiments has been suggested.

The positively charged clusters in the CuO-plane expel surrounding holes further away and reveal the background electrons. In our jellium approximation this shows up in the hole distribution function $g^{I}(r)$ as a deep minimum around the central hole peak extending to $2 r_{0}$ (see, Fig. 5). At infinity the distribution is normalized to one, which accounts for the fact that the charge of holes compensates totally the background charge and the integral of $g^{I}(r)-1$ is equal to the impurity charge.

\section{The dipolar clusters}

The cluster of four holes in the $\mathrm{CuO}$ plane self-bound due to many-body effects to the excess charge created in the $\mathrm{LaO}$ plane around the apex oxygen pushed by a dopant ion (the antiJahn-Teller effect) has a strong dipolar moment and low mobility. With increasing doping these clusters form a classical dipolar gas. The potential energy of $N$ dipoles is determined by the impurity charges $q_{\mathrm{LaO}}=-2|e|$ located in $\mathrm{LaO}$ layers, each of which creates the opposite sign charge cluster in a $\mathrm{CuO}$ layer $q_{\mathrm{CuO}}=2|e|$. For simplicity, we assume that these charges in both layers are point like, heavy objects and calculate their

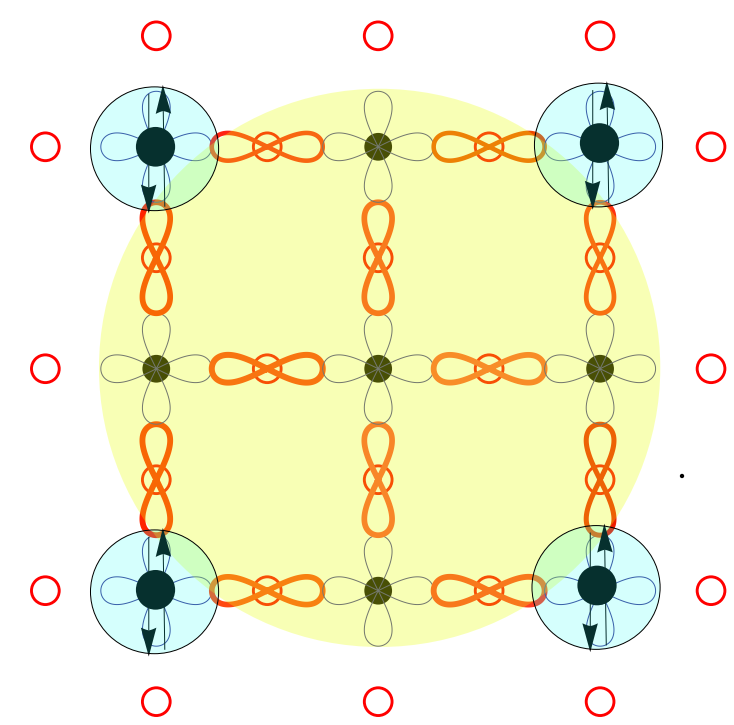

Figure 7: A schematic top view of the charged hole cluster in $\mathrm{CuO}$ plane of $\mathrm{La}_{2} \mathrm{CuO}_{4}$ noted by yellow disk. At the center of the cluster there is the $\mathrm{Cu}$ atom surrounded by four oxygen and other $\mathrm{Cu}$ atoms with the $\mathrm{d}$ and $\mathrm{p}$-state orbitals shown respectively. Inside the cluster there are four holes trapped. Outside double occupied states (Mottness) arising to keep the electro-neutrality.

Coulomb potential energy classically

$$
V=\frac{1}{4 \pi \epsilon} \sum_{i \neq j}^{N} \sum_{\alpha, \beta} \frac{q_{\alpha} q_{\beta}}{\left|\mathbf{r}_{\alpha}(i)-\mathbf{r}_{\beta}(j)\right|} .
$$

It depends on the charges and dielectric constant $\epsilon$, but most importantly it depends on the orientation of clusters. The Greek indices refer to the location of the charge, either in $\mathrm{LaO}$ or $\mathrm{CuO}$ layer, and $\mathbf{r}_{\alpha}(i)$ to the position of the charge in that layer. In the calculated examples we have required the following regular order. A bound dipole pair is separated by a distance $d$ pointing to the $b$ direction in the $a, b$-plane. The pairs are then separated by distances $R_{a}$ and $R_{b}$ in $a$ and $b$ directions, respectively. The motion of holes is strictly two-dimensional in the CuO-layers, but dopant atoms have some flexibility at high temperatures to

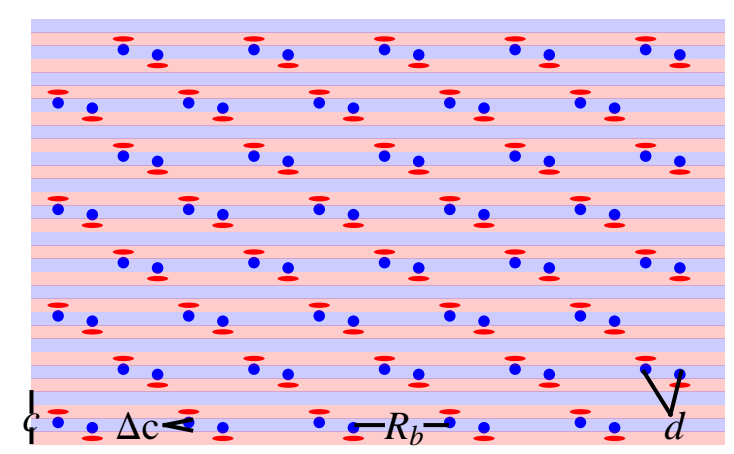

Figure 8: Ordered structure of dipolar clusters in the layered superconductors in the bc-plane. Four-hole clusters (red) are in the $\mathrm{CuO}$ plane and $\mathrm{i}-\mathrm{O}$ ions (blue) in $\mathrm{LaO}$ planes. The separation distance between $\mathrm{LaO}$ and $\mathrm{CuO}$ layers is $c / 4$. The separation distance between bound dipoles with opposite orientation is $d$ and the distance between dipole-dipole pairs is $R_{b}$. 


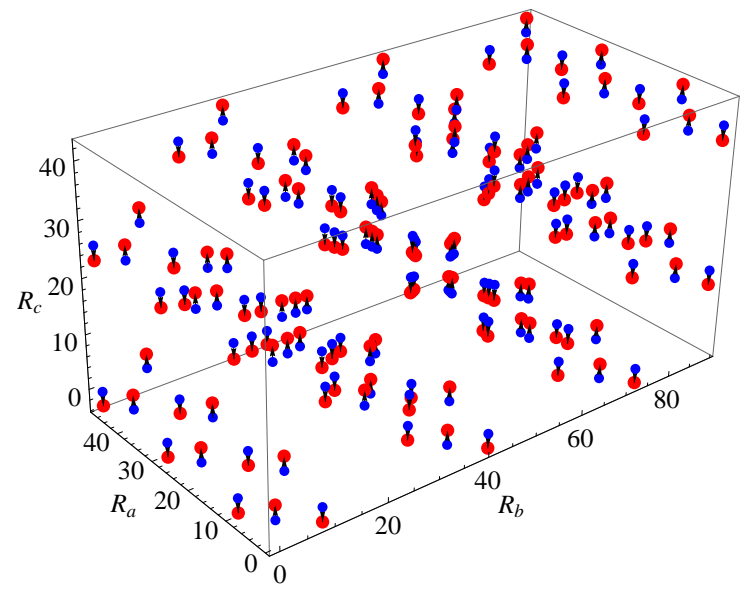

Figure 9: Regularly ordered clusters in 3D. The red circles determine the $\mathrm{CuO}$ plane. The i-O (blue circles) can be below or above that plane. The minimum energy in this configuration is reached when the dipole-dipole pairs are separated by $d=13 \AA$.

diffuse between two LaO layers. The octahedron can then be polarized either from above or below as shown in Fig. 8. With these assumptions the potential energy of two neighboring, oppositely oriented dipoles (d-d interaction) associated with the same $\mathrm{LaO}$ layer as a function of their separation distance $d$ is then

$$
V_{\mathrm{dd}}(d)=v(d, \Delta c)+v(d, c / 2)-2 v(d, c / 4+\Delta c / 2)
$$

where $v(d, x)=4 e^{2} /\left(4 \pi \epsilon \sqrt{d^{2}+x^{2}}\right), c=13.13 \AA$ is the size of the unit cell in c-direction and $\Delta c \approx c / 10=1.3 \AA$ is the separation distance of the impurity charge centers in c-direction as shown in Fig. 8. $V_{\mathrm{dd}}(d)$ has the minimum at $d=7.3 \AA$ with the energy $-12 \mathrm{meV}$, when we use the dielectric constant $\epsilon=21$ taken from our activation energy fit shown in Fig. 2. This defines the temperature scale in the self organization of the ordered structures seen in the experiments $[1,2]$.

The growth of nano grains under soft $\mathrm{X}$-ray radiation $[1,2]$ takes place in the temperature range, $330 \mathrm{~K}>\mathrm{T}>180 \mathrm{~K}$, well above the transition to superconducting state, but below the pseudogap where dipolar clusters exist. The $\mathrm{d}-\mathrm{d}$ interaction dominates the growth process. First appear bound d-d dimers in the $a-b$ plane and they arrange themselves into anti-ferroelectric stripes as shown in Fig. 9. The growth into larger clusters slows down the mobility and therefore patches of high density dipolar clusters can appear in different places of the sample. At high enough temperature depending on doping or hole density directional order melts and stripes disappear indicating a second phase transition inside the pseudogap region.

In Fig. 10 we show the contour plot of the potential energy in $\left(R_{a}, R_{b}\right)$ - plane for eight layers of $10 \times 10$ dipoles in a layer organized in a regular order like in Figs. 8 and 9. The dipoledipole separation distance $d=13 \AA$, which gives the minimum energy in this configuration. The energy surface has local minima and the growth process may not find the absolute minimum very easily. Fig. 11 then shows the potential energy per dipole as a function $R_{b}$, the d-d pair separation distance. We have fixed $d=13 \AA$ as above and $R_{a}=8.2 \AA$. The minimum energy is $-7 \mathrm{meV}$

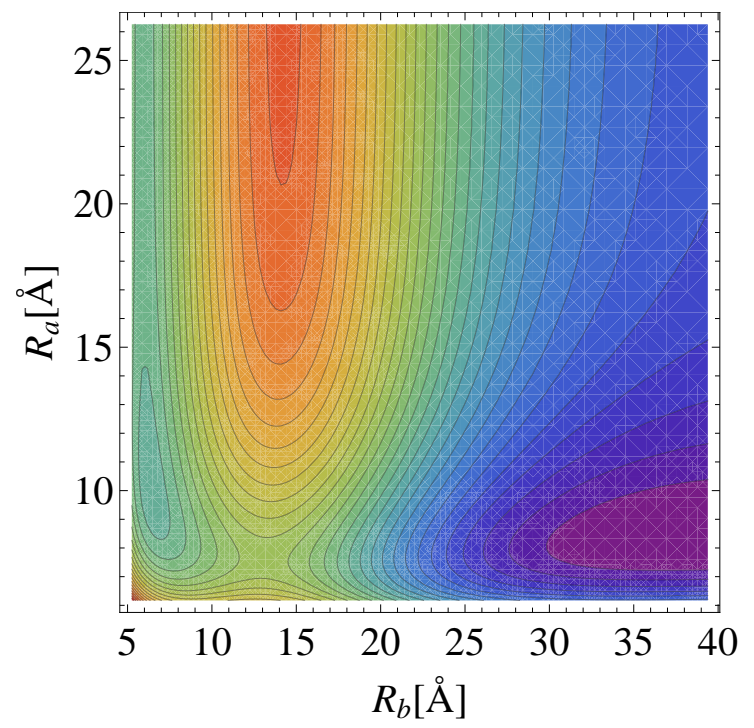

Figure 10: The contour plot of the potential energy per dipole in the $\left(R_{a}, R_{b}\right)$ -plane of regularly ordered clusters shown in Figs. 8 and 9. The third parameter in the potential energy of this ordered structure is the dipole-dipole separation distance $d$ in the $b$ direction, which has a fixed value $d=13 \AA$ obtained by minimizing the potential energy.

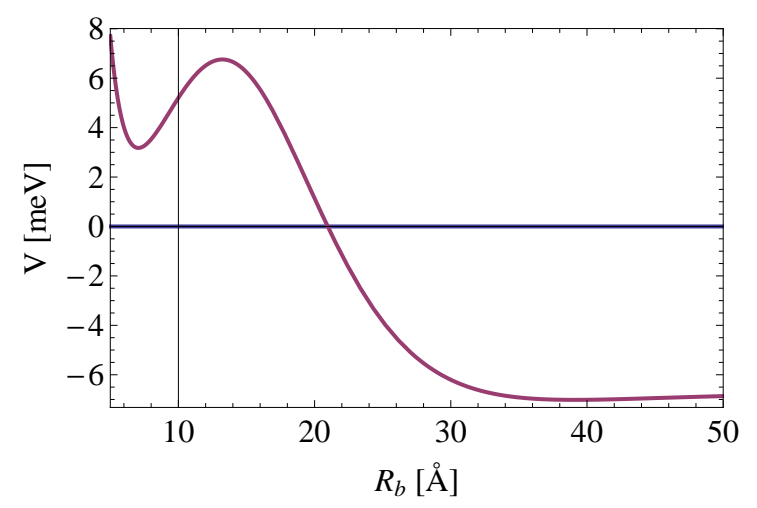

Figure 11: The potential energy per dipole of regularly ordered clusters shown in Figs. 8 and 9 as a function of separation distance between dipole-dipole pairs in $b$-direction $R_{b}$. Two other parameters have fixed values, $d=13 \AA$ and $R_{a}=8.2 \AA$

at $R_{b}=39 \AA$. This leads to a strong directional order and the system breaks into high and low density regions with different, nearly degenerate dipole orientations which grow slower and slower together but without the underlying, long-ranged crystal structure. Instead the system may favor the fractal like growth.

\section{Ferroelectricity at extremely low doping}

At extremely low doping, $x \approx 0.1 \%$, in $\mathrm{La}_{2-x} \mathrm{Sr}_{x} \mathrm{CuO}_{4}$ and $\mathrm{La}_{2} \mathrm{CuO}_{4-y}$ ferroelectricity has been discovered experimentally $[11,12]$. Measurements are done well inside the antiferromagnetic insulator phase and charge carrier holes are all localized. The dipolar structure between a dopant atom and clustered holes in $\mathrm{CuO}$ plane is strongly bound. In such dilute system distance $r$ between dopant atoms and, consequently, dipoles is large, $r \approx 30 \AA$. The dipole-dipole interaction dominating the 
ordered dipolar cluster formation diminishes like $1 / r^{3}$ and becomes very weak. Experiments in LCO $[1,2]$ suggest that the growth process of macroscopic dipolar structure under radiation takes place layer by layer. Thus in a very dilute system we may consider clustering of dipoles in separated layers. As discussed above dopant ions are located in spacer layers, push down apex oxygens and bind holes in $\mathrm{CuO}$ plane, say below the spacer layer. The dipole points to c-direction. The interaction with a neighboring dipole pointing to the same direction is repulsive. In order to gain energy dipoles must tilt away from cdirection more than the critical angle $\theta=\pi / 2-\arccos (1 / \sqrt{(3)})$. By tilting one increases the length of a dipole and the strength of the dipole moment, but looses in binding energy.

Experiments $[11,12]$ show that components of polarization in c- and ab-directions are roughly equal, which is consistent with the above estimate of the tilting angle. We can calculate the dipole-dipole binding energy $q^{2} /(4 \pi \epsilon) d^{2} / r^{3}\left(1-3 \cos ^{2}(\theta)\right)=$ $1.3 m e V(1-3 \cos (\theta))$, where $\epsilon=21 \epsilon_{0}$, the length of dipole $d=3 \AA$ and dipole separation distance $r=28 \AA$, ten times the distance between $\mathrm{Cu}$ atoms in $\mathrm{CuO}$-plane. This gives a rough idea of the temperature range of ferroelectric order to be below 6-7 K.

An important condition for ferroelectricity is the two-state structure. Direction of electric field favors parallel direction of dipoles by pushing dopant ions to one side of the spacer layer. Switching the direction of the external electric field pushes dopant ions to the other side of the spacer layer. Bound clusters in a CuO-layer are then created to the other side of the spacer layer, direction of dipoles is reversed and polarization charges sign. In doing this switching a typical hysteresis curve in the polarization-electric field plane should be seen. There must be a potential barrier in moving dopant ions yielding the hysteresis behavior. Experimentally the hysteresis loop is very thin suggesting that the potential barrier is low.

The energies of two dipole orientations $d_{1}$ and $d_{2}$ under the external electric fields $E_{1}$ and $E_{2}$ acting on them are $-\left(\mathbf{d}_{\mathbf{1}} \cdot \mathbf{E}_{\mathbf{1}}\right)$ and $-\left(\mathbf{d}_{\mathbf{2}} \cdot \mathbf{E}_{\mathbf{2}}\right)$, see Fig. 12. Therefore with each dopant ion we may introduce a partition function

$$
Z=\exp \beta\left(\mathbf{d}_{\mathbf{1}} \cdot \mathbf{E}_{\mathbf{1}}\right)+\exp \beta\left(\mathbf{d}_{\mathbf{2}} \cdot \mathbf{E}_{\mathbf{2}}\right)
$$

where $\beta=1 / k_{B} T$. The polarization associated with such a dopant ion $P_{i}$ can be estimated with the equation

$$
P_{i}=d_{1} \exp \beta\left(\mathbf{d}_{\mathbf{1}} \cdot \mathbf{E}_{\mathbf{1}}\right) / Z+d_{2} \exp \beta\left(\mathbf{d}_{\mathbf{2}} \cdot \mathbf{E}_{\mathbf{2}}\right) / Z
$$

Taking into account the staggered character of the intrinsic electrical field, we may introduce the fields $\mathbf{E}_{\mathbf{1}}=\mathbf{E}_{\text {int }}+\mathbf{E}$ and $\mathbf{E}_{\mathbf{2}}=-\mathbf{E}_{\text {int }}+\mathbf{E}$. Assuming that $\mathbf{d}_{\mathbf{2}}=-\mathbf{d}_{\mathbf{1}}=\mathbf{d}_{\mathbf{i}}$ we can write polarization in the following form,

$$
\mathbf{P}_{\mathbf{i}}=\mathbf{d}_{\mathbf{i}} \tanh \left(\beta \mathbf{E} \cdot \mathbf{d}_{\mathbf{i}}\right)
$$

In the regime of low doping total polarization $P$ of the crystal consists of contributions from all dopants ions. At very low temperatures $P=n_{i}<P_{i}>$ where $n_{i}$ is the density of dopant ions.

Next we develop a pseudo-spin formalism of this two-level system. The energy difference $2 \mathbf{E} \cdot \mathbf{d}_{\mathbf{i}}=h$ corresponds to an

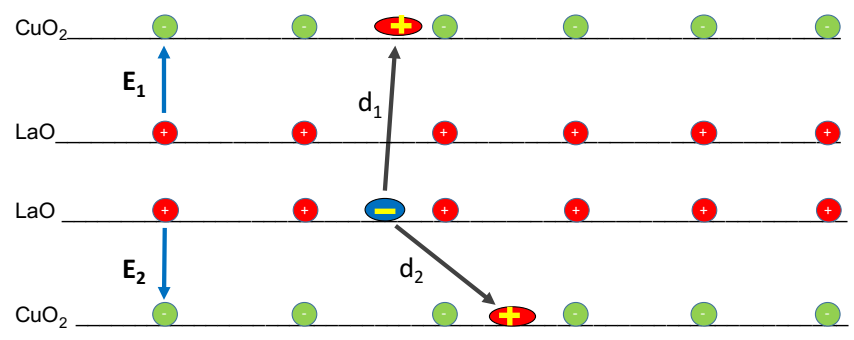

Figure 12: There is an intrinsic electric field between the $\mathrm{LaO}$ layers and $\mathrm{CuO} 2$ layers, It is noted as $E_{1}$ and $E_{2}$. These fields form a staggered layered structure over the crystal. The two states of the dipoles formed by a dopant atom located in the spacer layer, $\mathrm{LaO}$. The dipole may be located in the upper layer between $\mathrm{LaO}$ and $\mathrm{CuO} 2$, and its value is equal to $d_{1}$ or in the lower layer, below $\mathrm{LaO}$ where its value will be equal to $d_{2}$. Thus each dopant atom may be a sources of two states of a dipole.

effective field $h$. Without an interaction between dipoles thermodynamics of our model is simply equivalent to a standard behavior of non-interacting magnetic moments in an external field $h$. Therefore, the average "magnetization", which is here the polarization, associated with the pseudo-spins $\left\langle\mathbf{d}_{\mathbf{i}} \cdot \mathbf{E}\right\rangle$ is described by a standard Brillouin function for the spin $S=1 / 2$ as in Eq. (12).

The effective Hamiltonian describing interacting dipoles in the staggered electric field $E(z)$, may be written in the following form:

$$
H_{e f f}=\sum_{i j} \mathcal{J}_{\mathbf{i j}} \mathbf{d}_{\mathbf{i}} \otimes \mathbf{d}_{\mathbf{j}}-\sum_{i} \mathbf{E} \cdot \mathbf{d}_{\mathbf{i}}
$$

where the second term describes the interaction with staggered electric field as discussed above. The first term of the Hamiltonian is the complex interaction between dipoles and staggered electrical field associated with the charged layers $\mathrm{CuO}_{2}^{2-}$ and $\mathrm{LaO}^{+}$. It is represented with the use of the tensor $\mathcal{J}_{i j}^{\alpha \beta}$, which includes also the direct dipole-dipole interaction. In our situations of an interacting two level system shown in Fig. 12 the effective pseudo-spin Hamiltonian becomes

$$
H_{e f f}=\sum_{<i j>} \mathcal{J}_{i j} \tau_{i}^{z} \tau_{j}^{z}-h \sum_{i} \tau_{i}^{z}
$$

here we assume that there is an interaction between nearest two level systems in the sandwich of $\mathrm{CuO}_{2}^{2-}$ and $\mathrm{LaO}^{+}$pairs of layers. Note that we have introduced here the notation $\tau_{i}^{z} \equiv \mathbf{d}_{\mathbf{i}} \cdot \mathbf{E} /\left|\mathbf{d}_{\mathbf{i}} \cdot \mathbf{E}\right|$. One can easily show that although the coupling is a long-ranged in the first approximation we can take into account a nearest-neighbor interaction which can be both repulsive, $\mathcal{J}>1$, i.e. an antiferromagnetic interaction or attractive, $\mathcal{J}<1$, i.e. a ferromagnetic interaction between 
pseudospins $\tau$. In accordance with the qualitative considerations presented above (the state $\tau_{1}^{z}=+1$ corresponds to the up dipole orientations while $\tau_{1}^{z}=-1$ is the down dipole orientation. Longer range interactions may in general have different sign, but usually the $n n$ interactions dominate, and this is what we will assume further on.

With this assumption we can reduce our model to an antiferromagnetic or ferromagnetic Ising model with $n n$ coupling $\mathcal{J}$ in a parallel field. In this case the standard mean-field equation for the total magnetization takes the form:

$$
\tau=<\tau>=\tanh \frac{h-\mathcal{J} z \tau}{T}
$$

( $z$ is the number of nearest neighbours), from which we can determine the temperature dependence of $\tau$ and consequently the total polarisation of our system as $\mathbf{P}=<\mathbf{d}_{\mathbf{i}}>n_{\text {dip }}$.

It is convenient to rewrite Eq. (15) as

$$
\tau=\tanh \frac{\tilde{\Delta}+\mathcal{J} z(1-\tau)}{T}
$$

where $\tilde{\Delta}=h-\mathcal{J} z \tau(0)=h-\mathcal{J}_{z}$ is the renormalized initial $(T=0)$ splitting of these two states. If we would take this splitting due to $h$ to be constant (i.e. if we ignore the second term in the argument of Eq. (16), we would get the conventional temperature dependence of $\tau$ (Brillouin function) and, consequently, of the polarisation, which at low temperature would be exponential in temperature:

$$
\tau(T)=1-\exp \left(-\frac{\tilde{\Delta}}{T}\right)
$$

Eq. (15) can be solved numerically. It contains two parameters $h$ and $\mathcal{J} z$, which can be fit to experiments. Results are shown in Figs. 13 and 14 where the experimental data is taken from Fig. 2 of Ref. [11]. The value of $h=0.055 \mathrm{~K}$ is the same in both figures, but the interaction term changes slightly from $\mathcal{J} z=-6.9 \mathrm{~K}$ in the case of $P_{z}$ to $\mathcal{J} z=-6 \mathrm{~K}$ for $P_{a b}$. The interaction strength is consistent with the estimate of the dipole-dipole interaction given above. The over all strength of the polarization is fitted to the experimental value at $T=0$ to be equal $P_{z}(0)=37.8 \mathrm{nC} \mathrm{cm}{ }^{-2}$ and $P_{a b}(0)=18.7 \mathrm{nC} \mathrm{cm}^{-2}$. These values are defined by the total number of dipoles created in the samples. The figures show an excellent agreement between the developed theory and existing experiments. It is then clear that the critical temperature of the ferroelectric phase transition is determined by the dipole-dipole interaction at very low dopings, $x 0.1 \%$.

In Fig. 15 we show the behavior of the strength of polarization $P$ as a function of applied external field $E$ at the temperature $T=2 K$. Again we have a very good fit of our theory to the experiments of Ref. [11] for for the LCSO samples with very small doping $x=0.1 \%$. Polarization along the c-direction dominates due to the staggered field. Note also that the dependence of the in-plane polarization $P a b$ on applied electric field $E$, see the blue curve (the developed theory) and red dots (the experimental data from the Ref. [11] are well described by a standard Brillouin function for the spin $S=1 / 2$, which coincides with

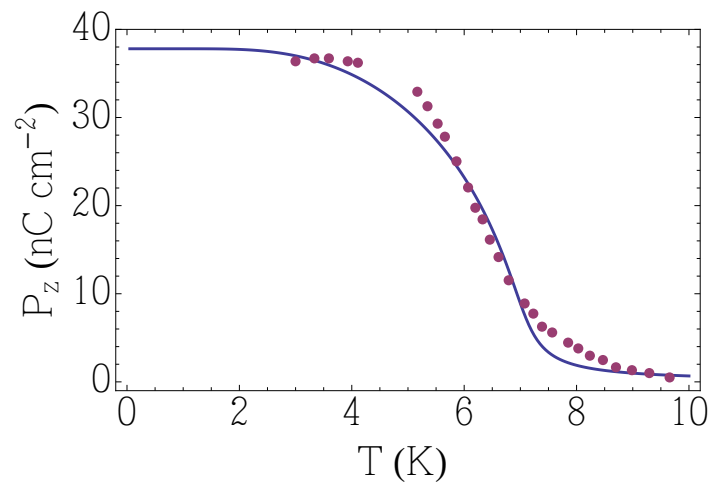

Figure 13: Out-of-plane $P_{z}$ electric polarization as a function of temperature $T$. Solid line corresponds to the theoretical calculation performed in the framework of the developed model, when the value of dipole interaction is chosen to be $\mathcal{J} z=-6.9 \mathrm{~K}$ and $h=0.055 \mathrm{~K}$. The dots correspond to experimental data taken from the paper[11].

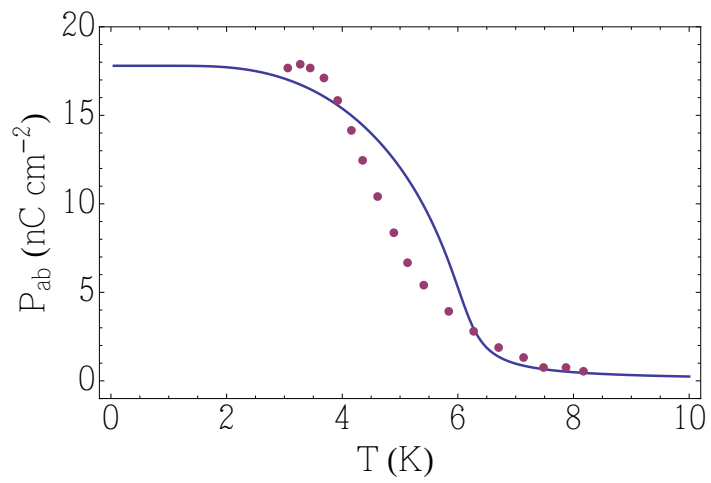

Figure 14: In-plane $P_{a b}$ electric polarization as a function of temperature. Solid line corresponds to the theoretical calculation performed in the framework of the developed model, when the value of dipole interaction is chosen to be $\mathcal{J} z=$ $-6.0 \mathrm{~K}$ and $h=0.055 \mathrm{~K}$. The dots correspond to experimental data taken from the paper[11].

the derived Eq. (12). However the similar dependence for the z-component of the polarisation, $P_{z}(E)$ shows already small deviation between the theory and experiments, that indicates that the dipole-dipole interaction should be here properly taken into account.

\section{The resonance plaquettes at optimal doping are viewed as Planckian dissipators}

For free hole current carriers each dipole is a source of strong scattering. The scattering becomes stronger when the doping increases and reaches its maximum at the optimal doping. To estimate the scattering time $\tau$ we have to look into the detailed structure of individual dipole clusters and their evolution with doping. Here each dipole is formed by a cluster of four holes located in the $\mathrm{CuO}$ plane and created by the many-body overscreening of the Coulomb attraction of these holes to the impurity or electron polarons located in the $\mathrm{LaO}$ spacer layer. The whole process of the cluster formation is accompanied by the squeezing of the neighbouring oxygen octahedron and creation of heavy polarons located in $\mathrm{LaO}$ spacer. That process of the 


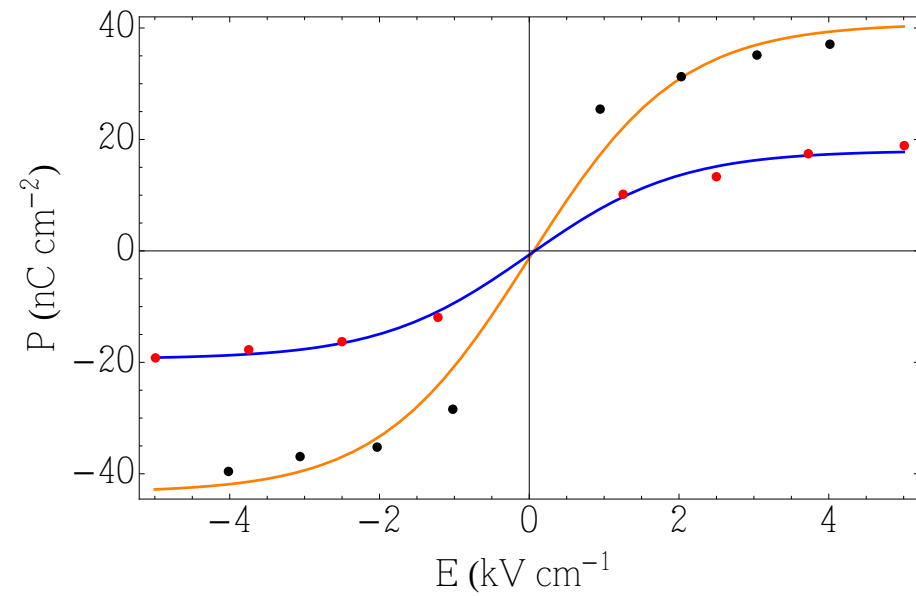

Figure 15: Polarization as a function of applied electric field (P-E) curves. The data for $P_{a b}$ (red solid circles) and for $P_{c}$ (black solid circles) were measured at $2 \mathrm{~K}$ and presented in the Ref. [11]. Blue and orange solid lines are calculated with the use of the derived Eq. (12). Here in the plot to fit the data the saturated $P_{z}$ was taken to be equal to $P_{z}=42 \mathrm{nC} \mathrm{cm}^{-2}$ and $P_{a b}=18.7 \mathrm{nC} \mathrm{cm}^{-2}$

oxygen octahedron squeezing is known as anti-Jahn-Teller effect and in turn it may provide four holes for the cluster and the singlet electron pair for the flat band as shown in Fig. 1. The rest doped free Zhang-Rice holes form a strongly-correlated quantum liquid. The many-body interaction of these holes in the strongly-correlated regime provides the over-screening of the charged impurities and polarons located in the LaO layer leads to the cluster formation. It is important to note that even without impurities the fluctuation associated with the squeezing of the oxygen octahedron locally, originated in such strongly correlated liquid may lead to formation of a similar cluster of four holes bound to polarons. However, such states are metastable and their structure depends on the doping level.

With increasing temperature clusters become metastable. The life time of such a fluctuating cluster, $\tau_{\text {life }} \sim \hbar / 2 E_{\text {bin }}(x)$, depends on its binding energy at a given doping $x$. It is maximal near optimal doping where $x \sim 0.2$. This effectively means that in the region of the optimal doping there are continuous quantum fluctuations associated with formation of these resonance plaquettes consiting of four holes. In other words they are decoupled from dopant impurities and become mobile.

Four holes clusters behave as quasi-classical objects moving along in a nearly flat band. Obviously such continuous fluctuations play huge role in the scattering of the remaining still very mobile free holes. As classical objects the thermal energy of clusters is $E_{k i n} \sim k_{B} T$. The motion of free current carriers through such a clustered media is very dissipative. Free holes can be individually trapped by cluster fluctuations and turn all their energy irreversibly into heat. Using the Heisenberg uncertainty principle we can estimate the scattering time of free holes as the time they leave in the cluster fluctuation, $E_{k i n} \tau \sim k_{B} T \tau \geq \hbar / 2$. Such a process takes a characteristic (relaxation) time,

$$
\tau=\tau_{\text {Planck }}=\hbar / 2 k_{B} T,
$$

which is the shortest possible relaxation time named as the
Planck time[41].

\section{Quantum Criticality and comparison with experiments}

The resistivity of the mixture of two components - free current carrier holes and four hole clusters - can be described by Drude formula, $R=m / n_{h}(x) e^{2} \tau$, where $m$ is the effective mass of holes and $n_{h}(x)$ is their density as a function of doping $x$. Near the optimal doping $x \sim 0.2$ the binding energy of clusters vanishes and fluctuation in the cluster formation will dominate the motion and transport of charge carriers. Inserting the scattering time of Eq. (18) into the Drude formula we get the linear T-dependent contribution to resistivity,

$$
R=\frac{2 m k_{B} T}{n_{h} e^{2} \hbar}=\alpha_{1}(x) T .
$$

In the underdoped region the binding energy of clusters increases with decreasing doping or increasing $r_{s}$ as shown in Fig. 2. Trapping of free holes into clusters becomes less probable and their transport becomes more Fermi liquid like with $T^{2}$ term in the resistivity.

We are now in position to make a comparison with experiments on LSCO $[25,26]$, where in the region of optimal doping the $\mathrm{T}$ - linear dependence of resistivity has been in detail investigated. The value of the effective mass $m=6.7 m_{e}$ and the hole density $n_{h}(x)$ are determined above from the comparison with the Hall coefficient measurements [29]. Inserting these numbers into Eq. (19) we get the T-linear coefficient. At $x=0.18$ $\alpha_{1}=1.14 \mu \Omega \mathrm{cm} / \mathrm{K}$ and at $x=0.21 \alpha_{1}=0.82 \mu \Omega \mathrm{cm} / \mathrm{K}$. These values are within the error bar of the experimental results[25], which is surprising for such an order of magnitude estimate. When $x<0.18$ the increasing binding energy of clusters diminishes the T-linear behavior and increses the normal Fermi liquid contribution as also seen in experiments [25].

These results confirm that the above theoretical calculations of the pseudogap (see, Fig.2) associated with the formation of the four holes clusters - the resonance plaquettes provide not only qualitative but also quantitative description of the underdoped and optimally doped cuprates, That also anticipates formation of anti-ferroelectric fractal structures which are all melted at optimally doping where a quantum phase transition arises.

\section{Discussion and Conclusions}

We have shown that in LSCO and other related compounds Coulomb interaction between charge carriers and doped ions is very important. The strong electronic correlations tightly connected with anti- Jahn-Teller lattice distortions lead to bound states of four holes forming a resonance plaquette. The formation of the plaquettes located in the $\mathrm{CuO}$ plane is associated with the over-screening of the Coulomb interaction. In the underdoped region they are bound with dopant atoms creating a cluster which has a dipole moment due to the separation of the plaquette and dopant impurity having opposite charges.

Recently Poccia et al. [3] proposed a mysterious second growth mechanism, which they have studied at $\mathrm{T}=85 \mathrm{~K}$. They 
relate that to the dynamics of local lattice distortion in the $\mathrm{CuO}$ plane. Also, in that case the scale free clusters, Q3, appear. The key question is what happens when the lattice is distorted and how does this distortion arise? The answer should be connected with the continuous X-ray illumination, which is a necessary condition for a growth of these snowflakes.

Indeed such an irradiation may create excitons of intermediate radius. In the Ref. [59] it was shown that if the excitons of intermediate radius are created they may create pairs of lattice defects as interstitial and vacancy. Moreover such excitons may have a radiationless decay into the pair consisting of the negatively charged oxygen interstitials, i-O, and the positively charged vacancy, $\mathrm{V}+$, which may be, in general, decoupled. Such excitonic mechanism of the local lattice defect formation and distortions has been observed experimentally in Refs. $[60,61]$, indeed. When such a vacancy will approach to another i-O, they may annihilate. The creation of such virtual or short time living pairs increases significantly the mobility of the i-O's and form as special pattern of the local lattice distortions, noted as Q3 in the Ref. [62, 3]. The pairs located in the LaO plane form dipoles lying in the plane that change the structure of the clusters. In fact there due to the presence of these extra coupled and decoupled i-O-V pairs the mobility of i-O's increase. This leads to a faster formation of the new type of clusters different from the ones described in the Refs. [1, 2, 63]. The additional dipoles associated with the new pairs created with $\mathrm{X}$-ray illumination lead to a new period of the stripe ordering in these new type of clusters noted as Q3 in the Ref. [59]. Besides the excitons generated the LLD-Q3 may arise only in the deeply underdoped case as excitons at all. The probability to such excitons with X-ray illumination very fast vanishes with the doping. This explains why the Q3 clusters are arising only in the underdoped cuprates.

Possibly, also the oxygen atoms may be displaced from their original positions in tetragons. They take one or two electrons with them, act as ions and leave behind holes, which are responsible of the superconducting state when the temperature is lowered below the critical temperature. These ions are not lifted into the $\mathrm{LaO}$ layer and are more mobile. However, negative ions form again bound states with holes and dipoles orient in the growth process as in the case of $\mathrm{i}-\mathrm{O}$ and form fractal clusters. The only difference between these two mechanisms is the location of the oxygen ions and shows up as slightly different stripe order of the nano grains.

Recently, the nature of oxygen dopant-induced states found at $-0.8 \mathrm{eV}$ in $\mathrm{Bi}_{2} \mathrm{Sr}_{2} \mathrm{CuO}_{8+x}$ by measuring ARPES spectra in a wide photon energy range has been investigated.[9] The found resonance profile of the corresponding nondispersive peak indicates an unexpected mixing with $\mathrm{Cu}$ states. The $\mathrm{A}_{1 g}$ symmetry of the peak suggests that the oxygen dopant-induced states are mixed with $\mathrm{Cu}$ through the $\mathrm{O}_{\text {dopant }}-\mathrm{O}_{\text {apex }} 2 \mathrm{p}_{z}-\mathrm{Cu} 3 \mathrm{~d}_{3 z^{2}-r^{2}}$ channel. [9]

Experiments indicate that the doped cuprates have a mixture of characters. To see this, we start with an undoped parent compound such as $\mathrm{La}_{2} \mathrm{CuO}_{4}$. This material is an insulator with a charge-transfer gap of $\sim 2 \mathrm{eV}$. Antiferromagnetism develops within the $\mathrm{CuO}_{2}$ planes as a consequence of strong on- site Coulomb repulsion between electrons in the same $\mathrm{Cu} 3 \mathrm{~d}$ $x^{2}-y^{2}$ orbital. The effective magnetic interaction is well characterized by the superexchange mechanism, and the magnetic excitation spectrum is described quite well by spin-wave theory with nearest-neighbor superexchange energy.[64]

Thus, the dipolar clusters appear when dopant oxygen ions in $\mathrm{LaO}$ layers trap charge carriers in $\mathrm{CuO}$ planes. The density of dipole moments increases with doping and vanishes at the pseudogap temperature. The binding energy of holes into these dipolar clusters defines the pseudogap, which we identify also as the activation energy of the two fluid model used by Gorkov and Teitelbaum. [30, 29, 57] Dipole moments have a strong interaction with electromagnetic radiation and subjected to such radiation dipoles become mobile. Due to the dipole-dipole attraction existing for some dipole orientations and repulsion for other orientations they may form clusters. This finding explains the recent observation of the self-organization of mobile oxygen dopant ions in LCO by Bianconi et al. [1, 2]. Under irradiation oxygen ions resting in the $\mathrm{LaO}$ layer as well as dipoles get excited and are forced to move towards the energetically most favourable positions.

Ferroelectricity in $\mathrm{La}_{2} \mathrm{CuO}_{4+y}$ and $\mathrm{La}_{2-x} \mathrm{Sr}_{x} \mathrm{CuO}_{4}$ was reported at exceptionally low doping.[11, 12]. We have shown that the dipole formation and its reduction to the two-level system under external electric explains the behavior of the ferroelectric polarization as a function of temperature and electric field. It could also be responsible of the peculiar behavior of the dielectric constant [10]. The resonance plaquettes described in this paper may play a very important role in the mechanism of high temperature superconductivity. Their presence in the vicinity of the hole band edge may lead to shape resonances as described recently by Bianconi[65], that can be an origin of the Cooper pairing in cuprates. Our results strongly suggest that many phenomena in cuprates such as pseudogap formation and high temperature superconductivity are interaction driven and therefore these materials belong to a new class of holographic superconductors[66].

\section{Acknowledgements}

We thank Antonio Bianconi, Lev Gorkov, Danya Khomskii, Christos Panagopoulos, Montu Saxena, Grisha Teitelbaum and Jan Zaanen for useful discussions. We also thank NanoSc-Cost Action MP1201 for financial support.

[1] M. Fratini, N. Poccia, A. Ricci, G. Campi, M. Burghammer, G. Aeppli, A. Bianconi, Nature 466 (2010) 841.

[2] N. Poccia, M. Fratini, A. Ricci, G. Campi, B. Luisa, A. Vittorini-Orgeas, G. Bianconi, G. Aeppli, A. Bianconi, Nature Materials 10 (2011) 733.

[3] N. Poccia, A. Ricci, G. Campi, M. Fratini, A. Puri, D. D. Gioacchino, A. Marcelli, M. Reynolds, M. Burghammer, N. L. Saini, G. Aeppli, A. Bianconi, Proceedings of the National Academy of Sciences 109 (39) (2012) 15685-15690. doi:10.1073/pnas.1208492109.

[4] P. Mallet, D. Roditchev, W. Sacks, D. Défourneau, J. Klein, Phys. Rev. B 54 (1996) 13324-13329.

[5] T. Cren, D. Roditchev, W. Sacks, J. Klein, Europhys.Lett. 54 (2001) 84.

[6] K. McElroy, J. Lee, J. A. Slezak, D.-H. Lee, H. Eisaki, S. Uchida, J. C. Davis, Science 309 (2005) 1048.

[7] I. Zeljkovic, Z. Xu, J. Wen, G. Gu, R. S. Markiewicz, J. E. Hoffman, Science 337 (6092) (2012) 320-323. 
[8] D. LeBoeuf, S. Kramer, W. N. Hardy, R. Liang, D. A. Bonn, C. Proust, Nat. Phys. 9 (2013) 79.

[9] P. Richard, Z.-H. Pan, M. Neupane, A. V. Fedorov, T. Valla, P. D. Johnson, G. D. Gu, W. Ku, Z. Wang, H. Ding, Phys. Rev. B 74 (2006) 094512.

[10] C. C. Wang, Y. M. Cui, G. L. Xie, C. P. Chen, L. W. Zhang, Phys. Rev. B $72(2005) 064513$.

[11] Z. Viskadourakis, S. S. Sunku, S. Mukherjee, B. M. Andersen, T. Ito, T. Sasagawa, C. Panagopoulos, Scientific Reports 5 (2015) 1. doi:10.1038/srep15268.

[12] Z. Viskadourakis, I. Radulov, A. P. Petrović, S. Mukherjee, B. M. Andersen, G. Jelbert, N. S. Headings, S. M. Hayden, K. Kiefer, S. Landsgesell, D. N. Argyriou, C. Panagopoulos, Phys. Rev. B 85 (2012) 214502.

[13] S. Mukherjee, B. M. Andersen, Z. Viskadourakis, I. Radulov, C. Panagopoulos, Phys. Rev. B 85 (2012) 140405.

[14] M. Hashimoto, I. M. Vishik, R.-H. He, T. P. Devereaux, Z.-X. Shen, Nat. Phys. 10 (2014) 483-495.

[15] A. Kaminski, T. Kondo, T. Takeuchi, G. Gu, Philosophical Magazine (2014) 1-14.

[16] T. J. Reber, N. C. Plumb, Y. Sun, Z .and Cao, Q. Wang, K. McElroy, H. Iwasawa, M. Arita, J. S. Wen, Z. J. Xu, G. Gu, Y. Yoshida, H. Eisaki, Y. Aiura, D. S. Dessau, Nat Phys 8 (606) (2012) 1-14.

[17] J. Zhao, U. Chatterjee, D. Ai, D. G. Hinks, H. Zheng, G. D. Gu, J.-P. Castellan, S. Rosenkranz, H. Claus, M. R. Norman, M. Randeria, J. C. Campuzano, Proceedings of the National Academy of Sciences 110 (44) (2013) 17774-17777.

[18] M. Hashimoto, E. A. Nowadnick, R.-H. He, I. M. Vishik, B. Moritz, Y. He, K. Tanaka, R. G. Moore, D. Lu, Y. Yoshida, M. Ishikado, T. Sasagawa, K. Fujita, S. Ishida, S. Uchida, H. Eisaki, Z. Hussain, T. P. Devereaux, Z.-X. Shen, Nat Mater 14 (2015) 37.

[19] T. T. Kondo, W. Malaeb, Y. Ishida, T. Sasagawa, H. Sakamoto, T. Takeuchi, T. Tohyama, S. Shin, Nat Commun 6 (2015) 1. doi:10.1038/ncomms8699.

[20] T. Wu, H. Mayaffre, S. Krmer, M. Horvati, C. Berthier, W. N. H. R. Liang, D. A. Bonn, M.-H. Julien, Nat Commun 6 (2015) 1.

[21] K. Fujita, C. K. Kim, I. Lee, J. Lee, M. H. Hamidian, I. A. Firmo, S. Mukhopadhyay, H. Eisaki, S. Uchida, M. J. Lawler, E.-A. Kim, J. C. Davis, Science 344 (6184) (2014) 612-616.

[22] A. Shekhter, B. J. Ramshaw, R. Liang, W. N. Hardy, D. A. Bonn, F. F Balakirev, R. D. McDonald, J. B. Betts, S. C. Riggs, A. Migliori, Nature 498 (2013) 75-77.

[23] R.-H. He, M. Hashimoto, H. Karapetyan, J. D. Koralek, J. P. Hinton, J. P. Testaud, V. Nathan, Y. Yoshida, H. Yao, K. Tanaka, W. Meevasana, R. G. Moore, D. H. Lu, S.-K. Mo, M. Ishikado, H. Eisaki, Z. Hussain, T. P. Devereaux, S. A. Kivelson, J. Orenstein, A. Kapitulnik, Z.-X. Shen, Science 331 (6024) (2011) 1579-1583.

[24] I. M. Vishik, M. Hashimoto, R.-H. He, W.-S. Lee, F. Schmitt, D. Lu, R. G. Moore, C. Zhang, W. Meevasana, T. Sasagawa, S. Uchida, K. Fujita, S. Ishida, M. Ishikado, Y. Yoshida, H. Eisaki, Z. Hussain, T. P. Devereaux, Z.-X. Shen, Proceedings of the National Academy of Sciences 109 (45) (2012) 18332-18337.

[25] R. A. Cooper, Y. Wang, B. Vignolle, O. J. Lipscombe, S. M. Hayden, Y. Tanabe, T. Adachi, Y. Koike, M. Nohara, H. Takagi, C. Proust, N. E. Hussey, Science 323 (2009) 603.

[26] N. BariÅi, M. K. Chan, Y. Li, G. Yu, X. Zhao, M. Dressel, A. Smontara, M. Greven, Proceedings of the National Academy of Sciences 110 (30) (2013) 12235-12240. doi:10.1073/pnas.1301989110.

[27] M. Först, A. Frano, S. Kaiser, R. Mankowsky, C. R. Hunt, J. J. Turner, G. L. Dakovski, M. P. Minitti, J. Robinson, T. Loew, M. Le Tacon, B. Keimer, J. P. Hill, A. Cavalleri, S. S. Dhesi, Phys. Rev. B 90 (2014) 184514.

[28] Y. Ando, Y. Kurita, S. Komiya, S. Ono, K. Segawa, Phys. Rev. Lett. 92 (2004) 197001

[29] S. Ono, S. Komiya, Y. Ando, Phys. Rev. B 75 (2007) 024515

[30] L. P. Gor'kov, G. B. Teitel'baum, Phys. Rev. Lett. 97 (2006) 247003.

[31] F. C. Zhang, T. M. Rice, Phys. Rev. B 37 (1988) 3759.

[32] Rykov, A. I., et al. "Change in the order of the melting transition with oxygen content in YBa 2 Cu 3 O 7? ?.” Physical Review B 60 (1999) 7601

[33] F. V. Kusmartsev, M. Saarela, Supercond. Sci. Technol. 22 (2009) 014008

[34] T. Tohayama, S. Maekawa, Supercond. Sci. Technol. 13 (2000) R17.

[35] T. Tohayama, S. Maekawa, Phys. Rev. B. 67 (2003) 092509.
[36] C. Kim, P. White, Z.-X. Shen, T. Tohayama, Y. Shibata. S. Maekawa, B. O. Wells, Y. J. Kim, R. J. Birgenau and A. Kastner, Phys. Rev. Lett. 80 (1998) 4245.

[37] P. W. Anderson, The Theory of Superconductivity in the High-Tc Cuprate Superconductors, Princeton University Press, Princeton, 1977.

[38] Kugel, K. I., et al. "A two-band model for the phase separation induced by the chemical mismatch pressure in different cuprate superconductors." Superconductor Science and Technology 22 (2008) 014007.

[39] G. Bednorz, A. Muller, Perovskite-type oxides -the new approach to hightc superconductivity, Nobel lecture. URL www.nobelprize.org/nobel-prizes/physics

[40] M. Saarela, F. Kusmartsev, Journal of Superconductivity and Novel Magnetism 28 (1) (2015) 1-5. doi:10.1007/s10948-014-2915-3.

[41] J. Zaanen, Nature 430 (2004) 512-513.

[42] F. V. Kusmartsev, Journal de Physique IV 9 (1999) 321-323.

[43] F. V. Kusmartsev, Phys. Rev. Lett. 84 (2000) 530-533.

[44] F. V. Kusmartsev, Phys. Rev. Lett. 84 (2000) 5026.

[45] M. Saarela, F. V. Kusmartsev, Phys. Lett. A 202 (1995) 317-323.

[46] M. Saarela, T. Taipaleenmäki, F. V. Kusmartsev, J. Phys. A 36 (2003) 9223.

[47] V. Apaja, S. Denk, E. Krotscheck, Phys. Rev. B 68 (2003) e195118.

[48] F. Kusmartsev, M. Saarela, Journal of Modern Physics B 29 (2015) $1542002,1-6$

[49] G. Bir, G. Pikus, Symmetry and strain induced effects in semiconductors, Wiley, New York 1974.

[50] V. J. Emery, Phys. Rev. Lett. 58 (1987) 2794.

[51] E. Feenberg, Academic, New York, 1969.

[52] T. Nishikawa, J. Takeda, M. Sato, Journal of the Physical Society of Japan 63 (4) (1994) 1441-1448.

[53] C. Y. Chen, et al, Phys. Rev. B 43 (1991) 392.

[54] T. Timusk, B. Statt, Rep. Prog. Phys. 62 (1999) 61.

[55] R. Yoshizaki, et al., Physica 166C (1990) 417.

[56] T. Nakano, et al., Phys. Rev. B 49 (1994) 16000.

[57] P. Phillips, Colloquium: Identifying the propagating charge modes in doped mott insulators, Rev. Mod. Phys. 82 (2010) 1719-1742.

[58] S. Chakraborty, P. Phillips, Phys. Rev. B 80 (2009) 132505.

[59] F. V. Kusmartsev, E. I. Rashba, Czech. J. Phys. 32 (1982) 54

[60] I. Fugol', Advances in Physics 37 (1) (1988) 1-35.

[61] E. Savchenko, Y. Rybalko, I. Fugol', Pis' ma Zh. Eksp. Teor. Fiz. 42 (5) (1985) 210-213.

[62] N. Poccia, A. Ricci, G. Campi, A.-S. Caporale, A. Bianconi, Journal of Superconductivity and Novel Magnetism 26 (8) (2013) 2703-2708. doi:10.1007/s10948-013-2164-x.

[63] P. Littlewood, Nature Materials 10 (2011) 726.

[64] J. M. Tranquada, Physica B: Condensed Matter 407 (11) (2012) 1771 1774.

[65] A. Bianconi, Nature Physics 9 (2013) 536-537.

[66] S. A. H. A. Donos, Nature Physics 9 (2013) 649-655. 